\title{
Incorporation of osteogenic and angiogenic small interfering RNAs into chitosan sponge for bone tissue engineering
}

This article was published in the following Dove Press journal:

International Journal of Nanomedicine

17 November 2014

Number of times this article has been viewed

\author{
Sen Jia ${ }^{1, *}$ \\ Xinjie Yangl,* \\ Wen Song $2, *$ \\ Lei Wang' \\ Kaixiu Fang ${ }^{3}$ \\ Zhiqiang $\mathrm{Hu}^{1,4}$ \\ Zihui Yang' \\ Chun Shan' \\ Delin Lei ${ }^{1}$ \\ Bin Lu'
}

'Department of Oral and Maxillofacial Surgery, ${ }^{2}$ Department of Prosthetic Dentistry, ${ }^{3}$ Department of Implant Dentistry, School of Stomatology, State Key Laboratory of Military Stomatology, Fourth Military Medical University, Xi'an People's Republic of China; ${ }^{4}$ Department of Otorhinolaryngology, No II 3 Hospital of People's Liberation Army, Ningbo, People's Republic of China

*These authors contributed to this paper equally and are considered to be joint first authors
Correspondence: Bin Lu, Delin Lei State key Laboratory of Military Stomatology, Department of Oral and Maxillofacial Surgery, School of Stomatology, Fourth Military Medical University, No. 145 West Changle Road, Xi'an 7I0032, People's Republic of China Email: lubinfxj@।63.com (Bin Lu); leidelin@fmmu.edu.cn (Delin Lei)

\begin{abstract}
Engineered bone substitutes are being extensively explored in response to growing demand. However, the angiogenesis that occurs during bone formation is often overlooked in scaffold design. In this novel study, we incorporated two small interfering RNAs (siRNAs), ie, small interfering RNA targets casein kinase 2 interaction protein 1 (siCkip-1) and small interfering RNA targets soluble VEGF receptor 1 (siFlt-1), which can promote osteogenesis and angiogenesis, into a chitosan sponge. This scaffold could maintain siRNAs for over 2 weeks in neutral phosphate-buffered saline and degraded rapidly in the presence of lysozyme. The chitosan sponge with siCkip-1 and siFlt-1 in vitro bioactivity was investigated using mesenchymal stem cells. Target genes were significantly suppressed, and osteocalcin, alkaline phosphatase, and vascular endothelial growth factor were significantly upregulated. Alizarin Red staining revealed that mineralization of the extracellular matrix was markedly enhanced by dual transfection. Further analysis by immunofluorescence confirmed that the siRNA-modified scaffold simultaneously improved the expression of osteocalcin and von Willebrand factor. In vivo testing in a skull critical-size defect model showed marked bone regeneration in rats treated with siCkip-1 and siFlt-1. In conclusion, chitosan sponge containing osteogenic and angiogenic siRNAs may be used as a scaffold for bone regeneration. The dual siRNA concept may also be useful in the biofunctionalization of other materials.
\end{abstract}

Keywords: chitosan sponge, osteogenesis, angiogenesis, small interfering RNA

\section{Introduction}

Defective bone can be a challenging problem in a number of settings, including trauma, deformity, infection, and cancer. ${ }^{1-3}$ Although the host may have the ability to recover, bone substitutes or grafts are required when the defects are larger than a critical size. ${ }^{4}$ Conventional efforts to improve the osteogenic potency of bone scaffolds have focused mainly on immobilizing growth factors to facilitate anabolic metabolism. ${ }^{5,6}$ Traditional tissue engineering scaffold-based implant materials focus mainly on effective and efficient osteogenesis; however, angiogenesis is another mandatory event during formation of bone that is often neglected in scaffold design. Angiogenesis and osteogenesis both require a blood supply that provides a sustentacular niche for selfrenewing osteoprogenitor cells and cytokines such as hypoxia inducible factor $1^{7}$ and vascular endothelial growth factor (VEGF) ${ }^{8}$ during bone formation. ${ }^{9}$ The coupling of angiogenesis and osteogenesis is regulated by a specific vessel subtype in bone. ${ }^{10}$ Consequently, an active blood vessel network is a prerequisite for bone regeneration. Combination therapies that promote both angiogenesis and osteogenesis during scaffold design and manipulation are necessary. ${ }^{11}$ 
RNA interference technology has provided a promising method to attenuate the production of specific target proteins by degradation of their mRNA. This powerful tool is being extensively studied as a potential treatment for numerous diseases, including cancer, ${ }^{12}$ viral infection, ${ }^{13}$ and neurodegenerative conditions. ${ }^{14}$ In recent years, small interfering RNA (siRNA)-modified scaffolds have also been widely explored for the purpose of engineering tissue regeneration by guiding multilineage differentiation of mesenchymal stem cells (MSCs) with incorporating siRNAs. ${ }^{15}$ It is considered to be a superior therapeutic strategy due to the high efficiency and targeting effect. Therefore, we hypothesize that incorporation of siRNAs that simultaneously promote osteogenesis and angiogenesis into the scaffold may solve the problem at the gene expression level during bone regeneration. Each type of siRNA has a clear specific target and the effect would be more precise and predictable compared with degradation of mRNA. Targeted knockdown of casein kinase 2 interacting protein 1 (Ckip-1) markedly improves the bone phenotype both in vitro and in vivo via the bone morphogenetic protein-related signaling pathway. ${ }^{16}$ Meanwhile, downregulation of soluble VEGF receptor 1 (sFlt-1) induces angiogenesis by release of additional free VEGF. ${ }^{17}$ Therefore, we delivered these two siRNAs simultaneously with the aim of promoting anabolic metabolism in defective bone.

A chitosan sponge was used as a scaffold to maintain and deliver the dual siRNAs. Chitosan sponges have been used widely as tissue engineering scaffolds in light of their simple fabrication, efficient aqueous absorption, and porous structure enabling cell penetration. ${ }^{18,19}$ These sponges can also release growth factors for bone regeneration in a controlled manner. ${ }^{20,21}$ To our knowledge, the present study is the first to use a chitosan sponge as a reservoir for two siRNAs. We analyzed this novel scaffold for bone regeneration in both in vitro and in vivo assays. Our results suggest that this siRNAmodified chitosan scaffold has marked beneficial effects on bone regeneration.

\section{Materials and methods Chitosan sponge preparation}

The chitosan sponge was prepared according to a previous report. ${ }^{12}$ Initially, $100 \mathrm{mg}$ of chitosan (molecular weight 100-300 kDa, degree of deacetylation 93.37\%; MP Biomedicals, Shanghai, People's Republic of China) was dissolved overnight in $10 \mathrm{~mL}$ of acetic acid $(2 \% \mathrm{v} / \mathrm{v})$ under magnetic stirring. The sticky chitosan solution was then put into a 24-well tissue culture plate at $1 \mathrm{~mL}$ per well.
The solution was frozen at a temperature below $-80^{\circ} \mathrm{C}$ for 4 hours, and the plate was then lyophilized for 24 hours. The lyophilized samples were neutralized by $1 \mathrm{M}$ sodium hydroxide and washed with deionized water until a neutral $\mathrm{pH}$ was reached. The hydrated samples were relyophilized using the same protocol.

\section{Loading of Lipofectamine ${ }^{\mathrm{TM}} 2000$ siRNA complexes}

To facilitate angiogenic differentiation, siRNA target rat sFlt-1(siFlt-1) ${ }^{13}$ was selected (sense 5'-GCGGGAGAGACUUAAACUATT-3'; antisense 5'-UAGuUuAaguCUCUCCCGCTT-3'; Shanghai GenePharma). To enhance osteogenic differentiation, siRNA target rat Ckip-1(siCkip-1) ${ }^{14}$ was chosen (sense 5'-GGACUUGGUAGCAAGGAAAdT*dT-3'; antisense 5'-UUCCUUGCUACCAAGUCCdT*dT-3'; Shanghai GenePharma, Shanghai, People's Republic of China). Universal negative control siRNA (sense 5'-UUCUCCGAACGUGUCACGUTT-3' and antisense 5'-ACGUGACACGUUCGGAGAATT-3'; Shanghai GenePharma) was used as a negative control (siNC). Transfection complexes were made by mixing $10 \mu \mathrm{L}$ of siFlt- 1 $(20 \mu \mathrm{M})$ and $10 \mu \mathrm{L}$ of siCkip-1 $(20 \mu \mathrm{M})$ with $500 \mu \mathrm{L}$ of $\alpha$-Minimum Essential Medium ( $\alpha$-MEM; Hyclone, Logan, UT, USA) or with $10 \mu \mathrm{L}$ of Lipofectamine 2000 (Invitrogen, Carlsbad, CA, USA) and $500 \mu \mathrm{L}$ of $\alpha$-MEM. After 5 minutes, the solutions were combined and added immediately to each well of the porous chitosan sponge. The siRNAloaded sponge was lyophilized again as described in chitosan sponge preparation section. The siNC was incorporated in a similar fashion.

\section{Degradation profile} for the siRNA-loaded scaffold

The siRNA-loaded sponge was immersed in either $500 \mu \mathrm{L}$ of phosphate-buffered saline or phosphate-buffered saline containing $0.1 \mathrm{~g} / \mathrm{L}$ lysozyme (Sigma-Aldrich, St Louis, MO, USA) for 28 days. At predetermined time points, samples of the solution were collected and replaced with fresh medium. The calibration curve was determined using standard siRNA, and the siRNA released in the collected samples was measured by RiboGreen (Invitrogen). The degradation rate was calculated by manually subtracting the siRNA released from the total siRNA loaded into the sponge. In addition, several selected sponges were freeze-dried during the degradation process and observed by scanning electron microscopy (SEM; Hitachi Ltd, Tokyo, Japan). 


\section{Cell culture}

Primary rat bone marrow-derived MSCs were isolated and cultured as described elsewhere. ${ }^{15}$ The animal experiments were approved by the animal welfare committee of the Fourth Military Medical University, Xi'an, People's Republic of China under the relevant laws and institutional guidelines. Briefly, male Sprague Dawley rats were sacrificed and sterilized in $75 \%$ ethanol for 15 minutes. The femurs were separated and bone marrow-derived MSCs were collected by douching with $\alpha$-MEM using a syringe under sterile conditions. Aspirates were centrifuged at $800 \mathrm{rpm}$ for 5 minutes, resuspended, and maintained in $\alpha$-MEM supplemented with $10 \%$ fetal bovine serum containing $100 \mathrm{U} / \mathrm{mL}$ penicillin and $100 \mu \mathrm{g} / \mathrm{mL}$ streptomycin. The medium was changed twice a week and passages 3-5 were used for further cell seeding. When $80 \%$ confluence was reached, the cells were trypsinized and centrifuged at $800 \mathrm{rpm}$ for 5 minutes. The pellet was suspended in medium to obtain a concentration of $2 \times 10^{5} / \mathrm{mL}$, and $1 \mathrm{~mL}$ was then added to the scaffold and placed in a 24-well plate. The scaffold was incubated for 2 hours to allow for adhesion, and another $1 \mathrm{~mL}$ of medium was then added.

\section{Cell attachment and proliferation}

Cell attachment was observed by SEM 1 day after cell seeding. Cells were fixed in $2 \%$ glutaraldehyde for 4 hours at $4^{\circ} \mathrm{C}$ and dehydrated using graded concentrations of ethanol. The sample was freeze-dried, coated with platinum, and observed. After different incubation times, cell proliferation was measured using a Cell Counting Kit (CCK-8; Beyotime Institute of Biotechnology, Jiangsu, People's Republic of China) according to the manufacturer's instructions. In brief, CCK- 8 and $\alpha$-MEM were mixed at a ratio of 1:10 and added to each well. After incubation for 3 hours, the supernatant was transferred to a 96-well plate and absorbance was read by a plate reader at $465 \mathrm{~nm}$.

\section{Colocalization of dual siRNAs and cells}

Cy3-labeled siCkip-1 (siCy3) and carboxyfluoresceinlabeled siFlt-1 (siFAM) were incorporated into the chitosan sponge. One day after cell seeding, the sample was fixed with 4\% paraformaldehyde (Sigma-Aldrich) for 20 minutes and cryosectioned by microtome in tissue freezing medium (Leica, Nussloch, Germany). The slices were then stained with 4,6-diamidino-2-phenyllindile (DAPI, Invitrogen) and observed by inverted fluorescence microscope (DMLRB, Leica). The cells from the scaffold were then trypsinized and centrifuged. The pellet was resuspended and checked by flow cytometry. Genome mean of red and green fluorescence intensity was used to represent the internalization efficiency.

\section{Real-time quantitative polymerase chain reaction}

Total RNA was isolated from the scaffold using a TRIzol ${ }^{\circledR}$ (Invitrogen)-based protocol. First, $1 \mu \mathrm{g}$ of total RNA was reverse-transcribed to complementary DNA by qScript ${ }^{\mathrm{TM}}$ complementary DNA (cDNA) SuperMix (Quanta Biosciences, Gaithersburg, MD, USA) according to the manufacturer's instructions. Real-time quantitative polymerase chain reaction (RT-qPCR) was performed on an $\mathrm{ABI}$ 7500 system with Platinum ${ }^{\circledR}$ SYBR ${ }^{\circledR}$ Green qPCR SuperMixUDG (Invitrogen). Normalized cDNA was amplified and the threshold cycle value was recorded by monitoring of fluorescence intensity. The relative mRNA level was calculated by the $\mathrm{Ct}$ value and normalized by glyceraldehyde-3phosphate dehydrogenase. The primer sequences are listed in Table 1.

\section{Immunofluorescence analysis}

The scaffold was maintained in normal medium for 3 days after seeding to allow for cell proliferation. Osteogenic induction was performed in an osteogenic medium container for 14 days. The scaffold was fixed in $4 \%$ paraformaldehyde for 20 minutes, then cryosectioned. The specimen was blocked by serum for 60 minutes and permeabilized with Triton ${ }^{\mathrm{TM}} \mathrm{X}-100$ (Invitrogen) for 15 minutes. The primary antibodies for anti-osteocalcin and anti-von Willebrand

Table I Primers used for real-time quantitative polymerase chain reaction

\begin{tabular}{|c|c|c|}
\hline Gene & Forward primer sequence $\left(5^{\prime}-3^{\prime}\right)$ & Reverse primer sequence $\left(5^{\prime}-3^{\prime}\right)$ \\
\hline Ckip-I & GAGCTTTCGGGTCGATCTGG & GGCTCCCTTGTCTGGTCTTT \\
\hline sFlt-I & TGACATACCCAAACTCGTG & AAAGCСTCTССTACTGTCC \\
\hline Alp & GGCTCCCTTGTCTGGTCTTT & GGACGCCGTGAAGCAGGTGA \\
\hline VEGF & CAGCTATTGCCGTCCAATTGA & CCAGGGCTTCATCATTGCA \\
\hline OCN & GGTGCAGACCTAGCAGACACCA & AGGTAGCGCCGGAGTCTATTCA \\
\hline GAPDH & CAAGTTCAACGGCACAGTCA & CCATTTGATGTTAGCGGGAT \\
\hline
\end{tabular}

Abbreviations: Ckip-I, casein kinase 2 interaction protein I; sFlt-I, soluble VEGF receptor I; Alp, alkaline phosphatase; VEGF, vascular endothelial growth factor; OCN, osteocalcin; GADPH, glyceraldehyde-3-phosphate dehydrogenase. 
factor was purchased from Abcam (Cambridge, MA, USA) and the specimens were incubated in those two antibodies respectively overnight. The specimens were incubated in fluorescein isothiocyanate-conjugated secondary antibody in the dark at room temperature for 1 hour. After rinsing with phosphate-buffered saline, the specimen was observed by fluorescence microscopy.

\section{Mineralization of the extracellular matrix} MSCs were seeded in the scaffold of a six-well plate. Osteogenesis-inducing medium containing 10\% fetal bovine serum, $5 \mathrm{mM}$ L-glycerophosphate, and $100 \mathrm{nM}$ dexamethasone (Sigma-Aldrich) and $50 \mu \mathrm{g} / \mathrm{mL}$ ascorbic acid was used for 14 days when confluence reached approximately $80 \%$. Cells in the scaffold were fixed in 10\% formalin and incubated with Alizarin Red S (Sigma-Aldrich, St Louis, MO, USA), $40 \mathrm{nM}, \mathrm{pH} 4.2$ ) solution for 10 minutes. The samples were rinsed thoroughly in deionized water and imaged by optical microscopy.

\section{Scaffold implantation and micro- computed tomography evaluation}

In vivo bone regeneration was evaluated using a rat calvarial bone defect model in which the rat was anesthetized using $1 \%$ pentobarbital, and a critical size defect of $8 \mathrm{~mm}^{16}$ was then created in calvarial bone followed by implantation of a chitosan-scaffold loaded with the different siRNAs. Three months later, the rats were sacrificed and the calvarial bone was fixed immediately in 4\% paraformaldehyde. The fixed samples were remodeled, inserted into the chamber, and scanned using an Inveon micro computed tomography (CT) system (Siemens AG, Munich, Germany). The region of interest was defined as a ring with a $4 \mathrm{~mm}$ radius from the center point of the defect. The three-dimensional structure of the defect and the newly-formed bone was reconstructed. The bone volume ratio was calculated by the software provided by the manufacture and used to represent the ratio of new bone mass.

\section{Van Gieson's stain}

The fixed samples were washed in running water, dehydrated by gradient ethanol $(50 \%-100 \%)$, and embedded in methylmethacrylate. Approximately $70 \mu \mathrm{m}$ thickness sections were obtained by hard tissue microtome (SP1600, Leica) paralleled to the long axis of the calvarial bone and running water cooled, slices approximately $70 \mu \mathrm{m}$ thick were harvested. The slices were polished and stained with $1 \%$ acid fuchsin and $0.5 \%$ saturated picric acid, respectively, for histological observation under a light microscope (Lucky Zoom, Olympus Corporation, Tokyo, Japan).

\section{Statistical analysis}

The statistical analysis was performed using Statistical Package for the Social Sciences version 17.0 software (SPSS Inc, Chicago, IL, USA). Three independent experiments were repeated in each section and the results are presented as the mean \pm standard error of the mean. One-way analysis of variance and the Student-Newman-Keuls $q$ test were used to compare means. $P<0.05$ was set as the level of statistical significance.

\section{Results \\ Characterization of siRNA-loaded sponges}

Under SEM, the formulated chitosan sponge was seen to be a porous three-dimensional structure with cracks approximately $100 \mu \mathrm{m}$ long and $50 \mu \mathrm{m}$ wide (Figure 1A). After loading with Lipofectamine/siNC, numerous Lipofectamine/siNC nanoparticles approximately $80 \mathrm{~nm}$ in size (Figure 1D) formed a larger aggregate approximately $10 \mu \mathrm{m}$ in size (Figure 1C) and became attached to the sponge (Figure 1B).

The degradation profile of the sponge was determined after 28 days. The siRNA was released slowly from the sponge during the first 8 days, with over $80 \%$ still left in the sponge (Figure 2A) at this time, which was released rapidly in the following 2 days. Thereafter, the siRNA was released at a steady rate until the final day, with about $15 \%$ remaining (Figure 2A) at that time. However, the siRNA was released far more rapidly (within 10 days) when in lysozyme solution and this is attributed to digestion of chitosan (Figure 2A). Meanwhile, chitosan dissolved in phosphate-buffered saline resulted in collapse and destruction of the cracks (Figure 2B1-B4). The same phenomenon was observed to occur more rapidly in phosphate-buffered saline and lysozyme solution (Figure 2B5-B6).

\section{Cell attachment and proliferation}

After 4 hours of attachment, the sphere-shaped cells were adsorbed loosely onto all of the scaffolds, with secretion of a small amount of extracellular matrix (Figure 3A). The MSCs proliferated rapidly during the first 3 days, but the rate of proliferation slowed thereafter. No significant difference in proliferation rate was observed between the sponges containing siCkip-1, siFlt-1, or siNC; however, the proliferation rate was significantly slower than on the sponge alone after 3 days (Figure 3B). 

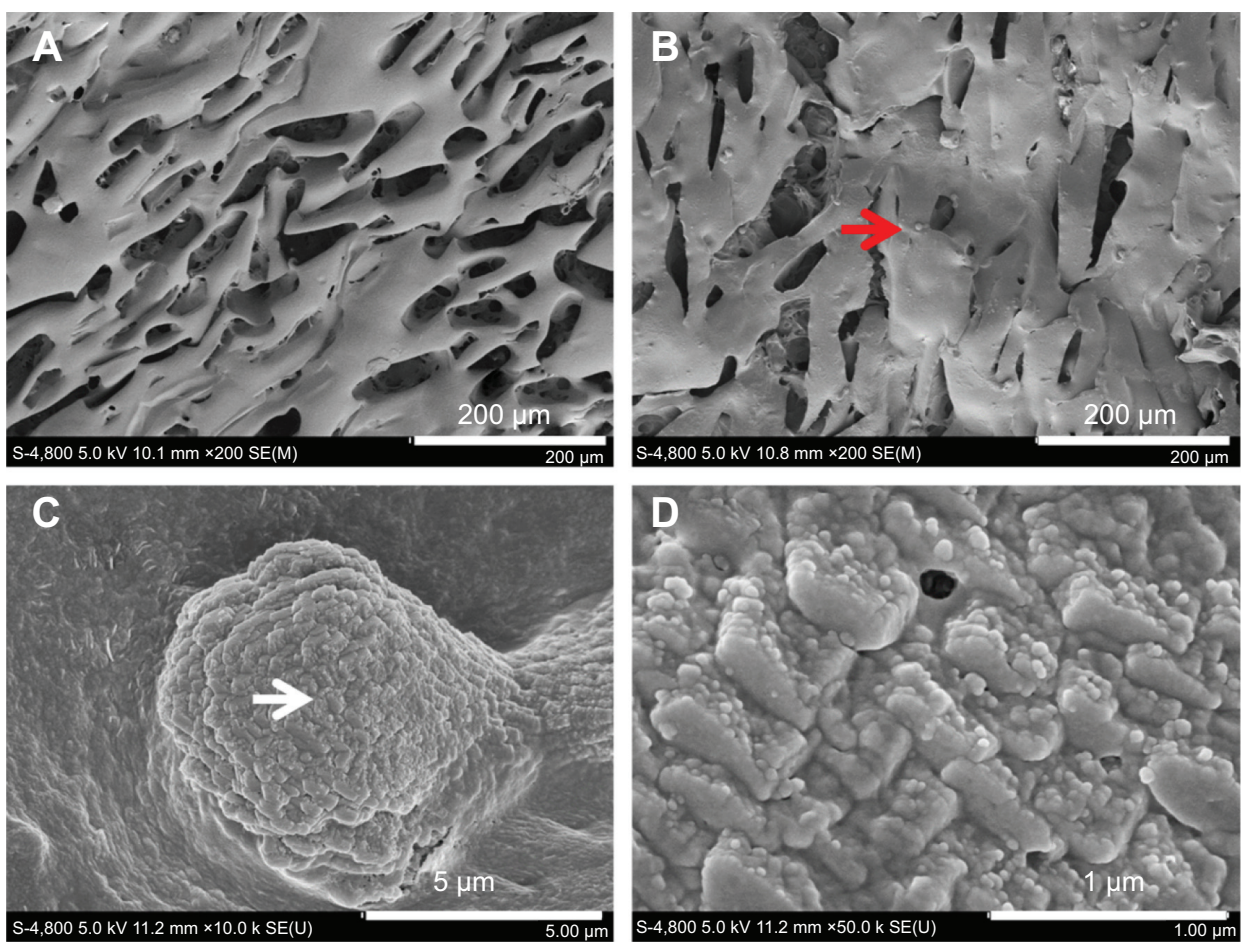

Figure I Morphology of chitosan sponge before and after loading with siRNA. (A) Blank chitosan sponge, (B) sponge loaded with siRNA, (C) high magnification of red arrow, and (D) high magnification of white arrow.

Abbreviation: siRNA, small interfering RNA.

\section{Colocalization of dual siRNAs and cells}

The sponge was cryosectioned to observe the uptake of dual siRNAs one day after cell seeding. It was obvious that Cy3-labeled red fluorescence and FAM-labeled green fluorescence were colocated with DAPI-stained blue nuclei, implying that the dual siRNAs could simultaneously be internalized into cells from the scaffold (Figure 4A). The internalization efficiency was further confirmed by flow cytometry. The sponge loaded with dual siRNAs showed at least a 5-fold increase in fluorescence intensity in both the red and green channels when compared with controls, which was similar to the counter mono siRNA internalization (Figure 4B).

\section{RT-qPCR analysis}

Relative gene expression was monitored by RT-qPCR. Sponges loaded with dual siRNAs could silence the both target genes $(-80 \%)$ on day 3 , which was almost the same level of the corresponding mono siCkip-1 or siFlt-1 transfection (Figure 5A). Due to the silencing of Ckip-1, mRNA for alkaline phosphatase and osteocalcin increased by $\sim 2.5$-fold in the dual transfection group and by $\sim 2$-fold in the mono siCkip-1

\section{A}

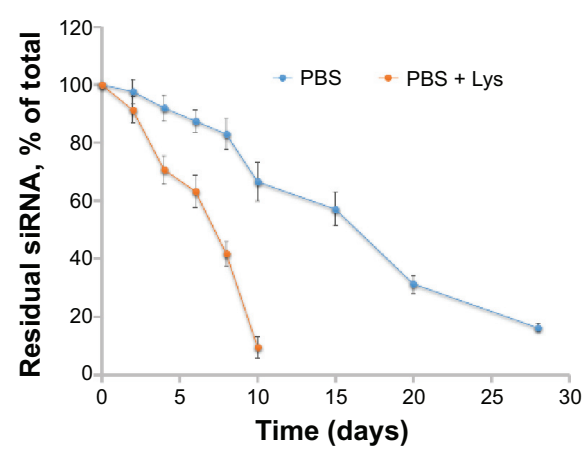

B
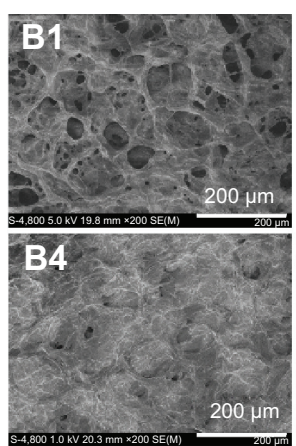
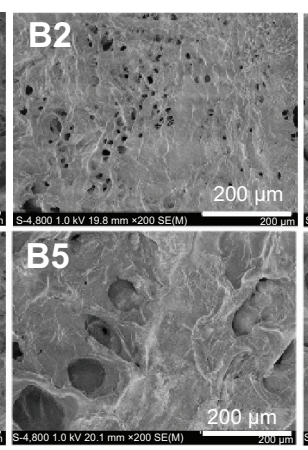

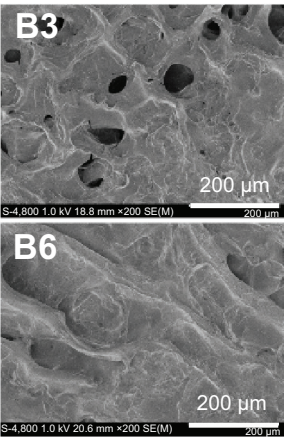

Figure 2 Degradation profile (A) and morphological observation of siRNA-loaded sponge either in PBS (B I-B4) or PBS+Lys (B5 and B6). Notes: B I-B4, incubation in PBS for 6, 10, 15 and 28 days respectively; B5 and B6, incubation in PBS+Lys for 6 and 10 days respectively. Abbreviations: PBS, phosphate-buffered saline; siRNA, small interfering RNA; Lys, lysozyme. 

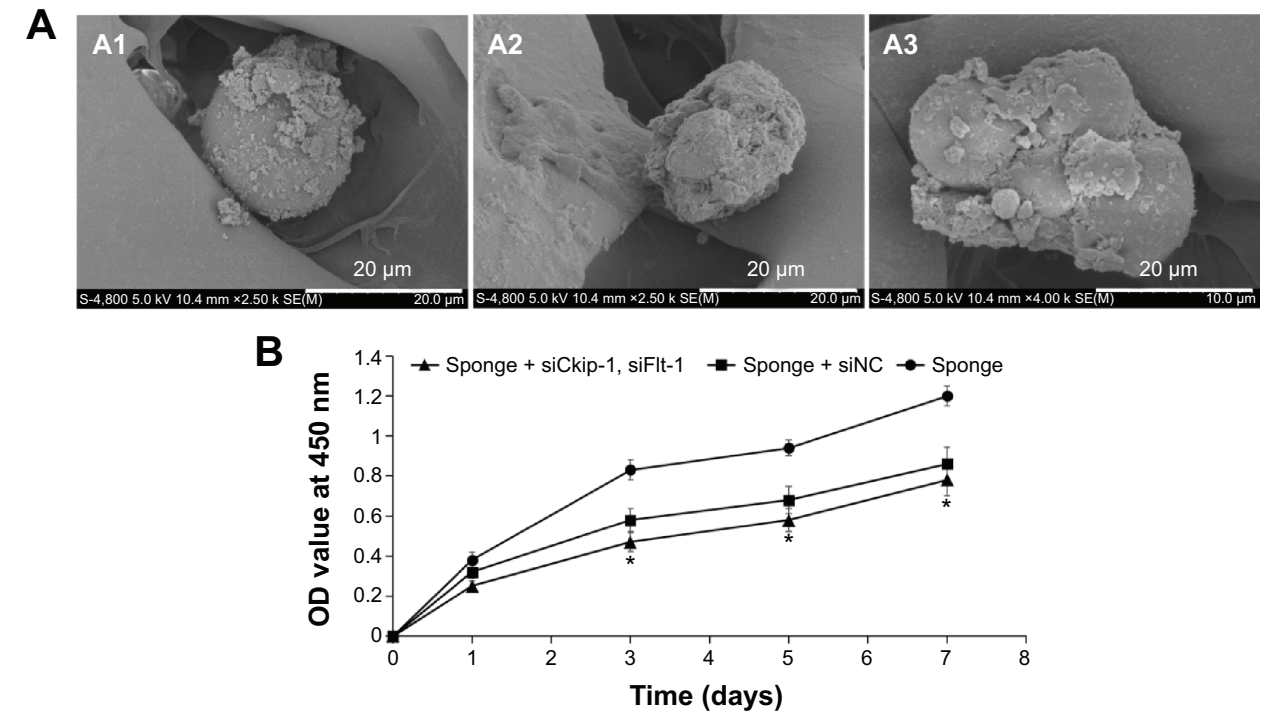

Figure 3 Cell attachment $(\mathbf{A})$ and proliferation (B) in the different scaffolds. (AI) Sponge + siCkip-I, siFlt-I, (A2) sponge + siNC, and (A3) sponge. Note: $* P<0.05$ versus sponge.

Abbreviations: OD, optical density; siNC, negative control small interfering RNA; siCkip-I, small interfering RNA targets casein kinase 2 interaction protein I; siFlt- I, small interfering RNA targets soluble VEGF receptor I.

group (Figure 5A). Similarly, VEGF increased markedly (by $\sim 3.5$-fold) in the dual transfection group and by $\sim 2.5$-fold in the mono siFlt-1 transfection group (Figure 5A). One week later, the target genes recovered to $\sim 50 \%$ in the sponge containing the dual siRNAs (Figure 5B). Alkaline phosphatase and osteocalcin increased by $\sim 5.5$-fold in the dual transfection group and by $\sim 3.5$-fold in the mono siCkip-1 transfection group (Figure 5B). Meanwhile, VEGF increased by 5fold in the dual transfection group and by $\sim 4.5$-fold in the mono siFlt-1 transfection group (Figure 5B). Interestingly, VEGF in the siCkip-1 mono transfection group increased by $\sim 2.5$-fold and alkaline phosphatase and osteocalcin by $\sim 2.5$-fold in the siFlt-1 mono transfection group, suggesting that crosstalk may exist (Figure 5B). This silencing effect disappeared after incubation for 2 weeks; however, alkaline phosphatase, osteocalcin, and VEGF expression was still over 3-fold higher in the dual siRNA group while the counter mono transfection groups were $\sim 2$-fold higher (Figure 5C). VEGF, alkaline phosphatase, and osteocalcin were also increased in the siCkip-1 and siFlt-1 mono transfection groups (Figure 5C).

\section{Mineralization in the extracellular matrix}

The osteoblast phenotype was confirmed by Alizarin Red staining for matrix mineralization. Osteogenic differentiation of MSCs was enhanced on either siCkip-1 or siFlt-1 loaded
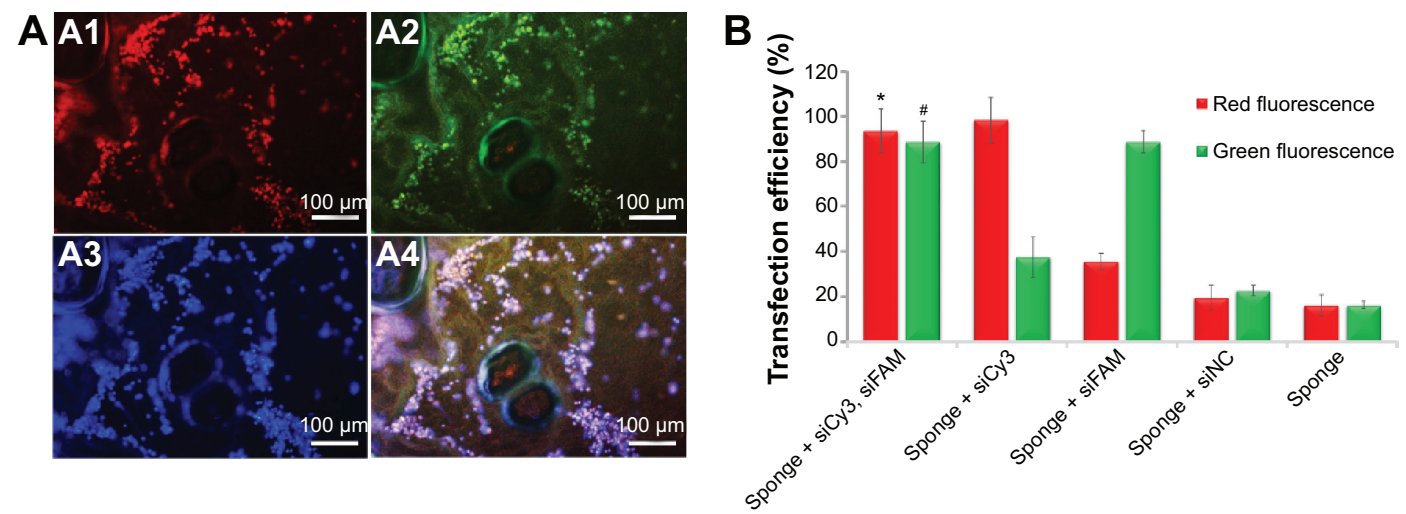

Figure 4 Colocalization analysis by fluorescence microscopy (A) and flow cytometry (B). (AI) Cy3-labeled siCkip-I, (A2) FAM-labeled siFlt-I, (A3) DAPI-stained nucleus, (A4) overlay of $\mathrm{A} 1, \mathrm{~A} 2$, and $\mathrm{A} 3$. $* \mathrm{P}<0.05$ versus siFAM, siNC, or sponge alone.

Note: ${ }^{\#} P<0.05$ versus siCy3, siNC, or sponge alone.

Abbreviations: DAPI, 4,6-diamidino-2-phenyllindile; siNC, negative control small interfering RNA; siCkip-I, small interfering RNA targets casein kinase 2 interaction protein I; siFIt-I, small interfering RNA targets soluble VEGF receptor I; siFAM, small interfering RNA targets FAM; siCy3, small interfering RNA targets Cy3. 
A

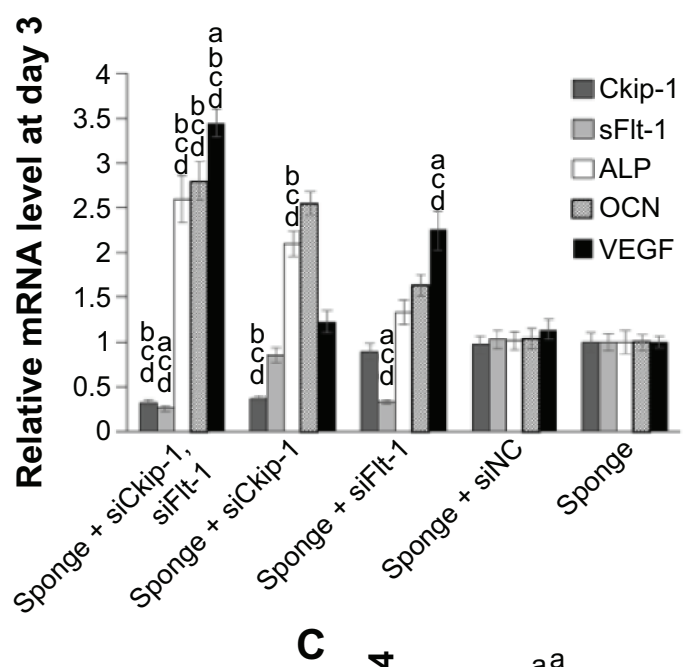

C
B

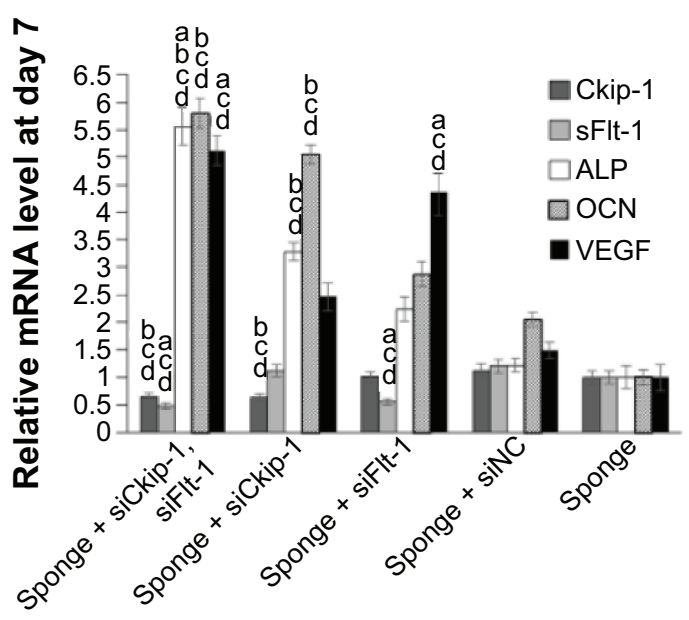

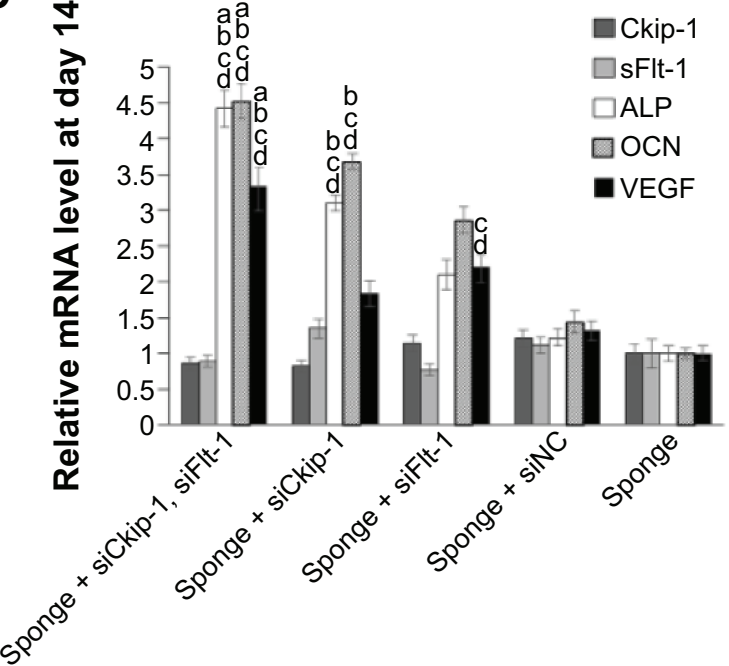

Figure 5 Gene expression measured by real-time quantitative polymerase chain reaction after incubation for 3 days (A), 7 days (B), and I4 days (C),

Notes: ${ }^{\mathrm{a}-\mathrm{d}} \mathrm{P}<0.05$ versus mono siCkip-I, siFlt-I, and siNC transfection or blank sponge, respectively.

Abbreviations: ALP, alkaline phosphatase; OCN, osteocalcin; VEGF, vascular endothelial growth factor; siNC, negative control small interfering RNA; siCkip-I, small interfering RNA targets casein kinase 2 interaction protein I; siFIt-I, small interfering RNA targets soluble VEGF receptor I.

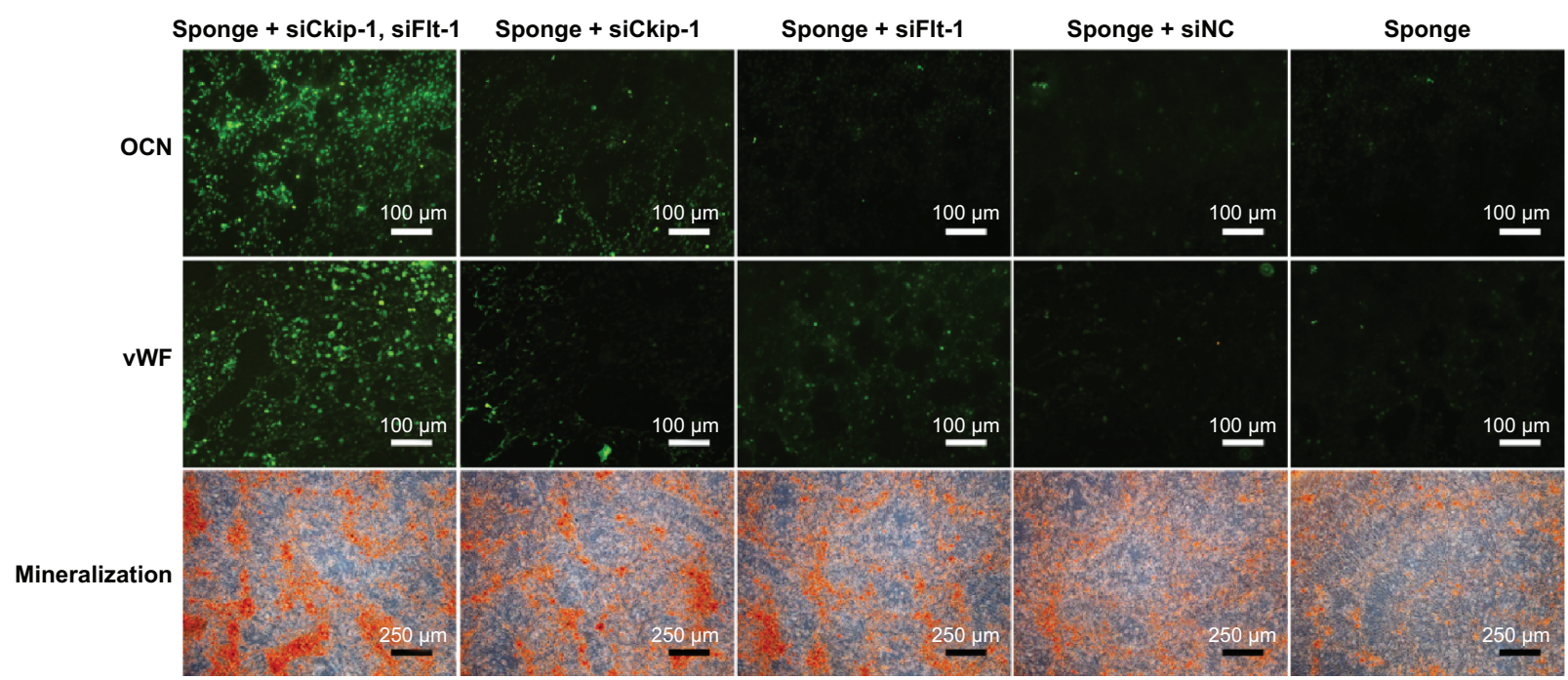

Figure 6 In vitro osteogenic differentiation analyzed by immunofluorescence and Alizarin Red staining.

Abbreviations: OCN, osteocalcin; vWF, von Willebrand factor; siNC, negative control small interfering RNA; siCkip-I, small interfering RNA targets casein kinase 2 interaction protein I; siFIt-I, small interfering RNA targets soluble VEGF receptor I. 
sponge compared with siNC or blank sponge (Figure 6). However, sponge loaded with both siCkip-1 and siFlt-1 could further increase the osteogenic differentiation when compared with the group loaded with siCkip-1 or siFlt-1 (Figure 6).

\section{Immunofluorescence assay}

The in vitro bone anabolic potency of the scaffold was determined by immunofluorescence staining. Osteocalcin was abundantly expressed on the dual siRNA-loaded scaffold but remained at a low level in the other scaffolds (Figure 6). In addition, the novel vascularization protein, von Willebrand factor, was also markedly enhanced on the dual siRNAmodified scaffold (Figure 6). As expected, osteocalcin was also upregulated in the mono siCkip-1 transfection group, as was von Willebrand factor in the mono siFlt-1 transfection group (Figure 6).

\section{Bone regeneration in the rat skull defect model}

New bone formation in the defect area was scanned by microCT and the bone volume ratio in the region of interest was taken to represent newly generated bone. The sponge loaded with dual siRNAs was able to promote significant defect restoration when compared with the other groups
(Figure 7A and C). Mono siCkip-1 transfection could also increase new bone formation when compared with the siNC or blank sponge. However, mono siCkip-1 transfection improved new bone formation much less in comparison with dual transfection (Figure 7A and C). The sectioned sample was stained according to Van Gieson's protocol after ultrahard slicing. As with microCT scanning, the twodimensional new bone area within the defect was largest in the dual transfection group; mono siCkip-1 also promoted more new bone generation in comparison with the siNC or blank sponge (Figure 7B and D).

\section{Discussion}

In this study, siCkip-1 and siFlt-1 were coimmobilized into chitosan to form a novel scaffold. These two siRNAs could be maintained in this scaffold for over 2 weeks and were able to colocate around seeded MSCs. In vivo and in vitro tests demonstrated that chitosan sponge containing osteogenic and angiogenic siRNAs promoted significant regeneration of bone. The present study may provide a new treatment strategy for bone defect.

Dual delivery of two therapeutic molecules can have simultaneous functional effects, with a synergistic outcome. $\mathrm{Qu}$ et $\mathrm{al}^{17}$ used basic fibroblast growth factor, which has activity in both osteogenesis and angiogenesis, to treat bone
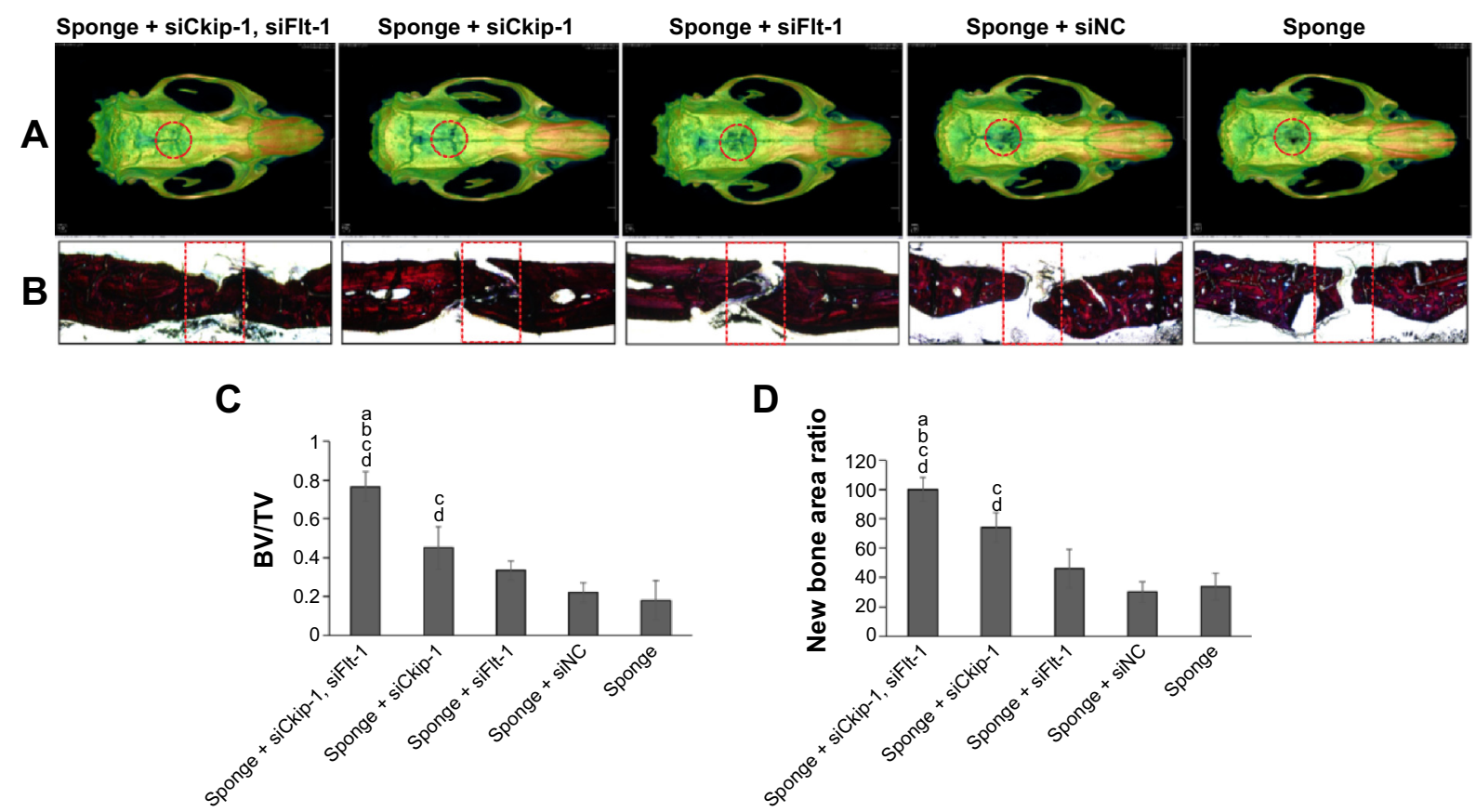

Figure 7 Bone regeneration in the rat skull defect model. (A) microCT reconstruction and the red circle represent the region of interest. (B) Van Gieson's staining and the red rectangle represents the region of interest. (C) New bone volume ratio. (D) New bone area ratio.

Notes: ${ }^{\mathrm{a}-\mathrm{d} P}<0.05$ versus mono siCkip-I, siFlt-I, and siNC transfection or blank sponge, respectively.

Abbreviations: BV, bone volume; TV, total volume; siNC, negative control small interfering RNA; siCkip-I, small interfering RNA targets casein kinase 2 interaction protein I; siFIt-I, small interfering RNA targets soluble VEGF receptor I. 
MSCs by seeding this factor into a nanohydroxyapatite/ polyamide66 (n-HA/PA66) composite scaffold to mimic natural osteogenesis. ${ }^{17} \mathrm{~B}$-cell lymphoma-extra large siRNA and doxorubicin have been codelivered for a synergistic anticancer effect. ${ }^{18}$ Cao et al have also synthesized and assembled biodegradable nanocarriers for dual delivery of siRNA and chemotherapeutic drugs into hepatic cancer cells. ${ }^{19}$ Moreover, siRNAs have been transfected to modulate the T-cell response by dual mode. ${ }^{20}$ Unfortunately, dual delivery of two siRNAs in bone tissue engineering is still seldom validated.

In our study, siRNAs promoting osteogenesis and angiogenesis were codelivered by Lipofectamine derived from chitosan sponge. Chitosan is an inexpensive and nontoxic polymer which has been extensively used as a drug carrier system. ${ }^{21}$ Chitosan sponge is a porous and plastic material compared with solid bone substitutes, so it is favorable for both drug loading and irregular bone defects restoration. Therefore, it may be more suitable for craniomaxillofacial defects regeneration since most of the bones here are curved and complicated. ${ }^{22}$ In addition, chitosan sponge can be synthesized without additional reagents that could have unwanted effects. For these reasons, chitosan sponge was used as both a drug reservoir and regeneration material in our study.

Chitosan sponge has been used as a carrier enabling sustained drug release. ${ }^{21}$ Our results confirmed that two siRNAs can have a superimposed action when cotransfected into MSCs from the sponge. In the surface characterization study, numerous Lipofectamine/siRNA particles aggregated together after lyophilization; ${ }^{23}$ however, the particles behaved very differently when compared with those from the direct measurement from aqueous solution. ${ }^{23}$ This aggregation might possibly be ascribed to the use of a lyophilization process without nonionic lyoprotectants. ${ }^{24}$ The siRNA release from the sponge could persist for a relatively long duration, which could probably acquire prolonged gene silence effect. In addition, the RT-qPCR data showed that the target genes were suppressed for at least 1 week. The growth rates of MSCs in the sponge + siCkip-1, siFlt-1, and sponge + siNC groups were not significantly different; however, both of them significantly lower than the sponge alone, indicating that the suppression may have originated from Lipofectamine and it was considered as its main drawback..$^{22,25,26}$ Colocalization observed by fluorescence microscope and quantitative data with flow cytometer assay showed high transfection efficiency in MSCs, which is in agreement with a previous report, ${ }^{22}$ and suggests that Lipofectamine could be used to deliver two siRNAs simultaneously. These results indicate that the chitosan sponge can be used to deliver dual siRNAs in a sustainable manner.

siCkip-1 is a well-documented anabolic molecule in bone ${ }^{14}$ and the increased VEGF caused by siFlt-1 might also improve bone formation. ${ }^{27}$ Similarly, VEGF expression was improved after silencing of Ckip-1. Therefore, knockdown of Ckip-1 or sFlt-1 alone was able to increase the expression of osteogenic genes and new bone regeneration to some degree. However, when they were delivered together, osteogenic differentiation was markedly improved both in vitro and in vivo, indicating that the angiogenic effect of siFlt-1 could further enhance the osteogenic effect of siCkip-1. Taken together, manipulating osteogenesis and angiogenesis simultaneously during bone tissue engineering may provide a more powerful efficiency. $^{28}$

\section{Conclusion}

siRNA-modified chitosan sponges could maintain siRNA for a longer term without losing its efficacy. Chitosan sponges loaded with both siCkip-1 and siFlt-1 had superimposed activity, resulting in a high degree of colocalization with cells and simultaneous enhancement of both osteogenesis and angiogenesis in vitro. Our in vitro and in vivo studies show that this scaffold is able to promote new bone regeneration. This dual siRNA-modified chitosan scaffold could potentially be used for restoration of healthy bone.

\section{Disclosure}

The authors report no conflicts of interest in this work.

\section{References}

1. Wiese A, Pape HC. Bone defects caused by high-energy injuries, bone loss, infected nonunions, and nonunions. Orthop Clin North Am. 2010; 41(1): $1-4$.

2. Nishida J, Shimamura T. Methods of reconstruction for bone defect after tumor excision: a review of alternatives. Med Sci Monit. 2008;14(8): RA107-RA113.

3. Ueda K, Oba S, Omiya Y, Okada M. Cranial-bone defects with depression deformity treated with ceramic implants and free-flap transfers. Br J Plast Surg. 2001;54(5):403-408.

4. Gomes PS, Fernandes MH. Rodent models in bone-related research: the relevance of calvarial defects in the assessment of bone regeneration strategies. Lab Anim. 2011;45(1):14-24.

5. Berner A, Boerckel JD, Saifzadeh S, et al. Biomimetic tubular nanofiber mesh and platelet rich plasma-mediated delivery of BMP-7 for large bone defect regeneration. Cell Tissue Res. 2012;347(3):603-612.

6. Bae SE, Choi J, Joung YK, Park K, Han DK. Controlled release of bone morphogenetic protein (BMP)-2 from nanocomplex incorporated on hydroxyapatite-formed titanium surface. J Control Release. 2012;160(3): 676-684.

7. Mamalis AA, Cochran DL. The therapeutic potential of oxygen tension manipulation via hypoxia inducible factors and mimicking agents in guided bone regeneration. A review. Arch Oral Biol. 2011;56(12):1466-1475.

8. Feng L, Wu H, E L, et al. Effects of vascular endothelial growth factor 165 on bone tissue engineering. PLoS One. 2013;8(12):e82945. 
9. Towler DA. The osteogenic-angiogenic interface: novel insights into the biology of bone formation and fracture repair. Curr Osteoporos Rep. 2008;6(2):67-71.

10. Kusumbe AP, Ramasamy SK, Adams RH. Coupling of angiogenesis and osteogenesis by a specific vessel subtype in bone. Nature. 2014;507(7492): 323-328.

11. Kanczler JM, Oreffo RO. Osteogenesis and angiogenesis: the potential for engineering bone. Eur Cell Mater. 2008;15:100-114.

12. Noel SP, Courtney HS, Bumgardner JD, Haggard WO. Chitosan sponges to locally deliver amikacin and vancomycin: a pilot in vitro evaluation. Clin Orthop Relat Res. 2010;468(8):2074-2080.

13. Ahmad S, Hewett PW, Al-Ani B, et al. Autocrine activity of soluble Flt-1 controls endothelial cell function and angiogenesis. Vasc Cell. 2011; 3(1):15.

14. Zhang G, Guo B, Wu H, et al. A delivery system targeting bone formation surfaces to facilitate RNAi-based anabolic therapy. Nat Med. 2012;18(2):307-314.

15. Song W, Wu K, Yan J, Zhang Y, Zhao L. MiR-148b laden titanium implant promoting osteogenic differentiation of rat bone marrow mesenchymal stem cells. RSC Adv. 2013;3:11292-11300.

16. Spicer PP, Kretlow JD, Young S, Jansen JA, Kasper FK, Mikos AG. Evaluation of bone regeneration using the rat critical size calvarial defect. Nat Protoc. 2012;7(1):1918-1929.

17. Qu D, Li J, Li Y, et al. Angiogenesis and osteogenesis enhanced by bFGF ex vivo gene therapy for bone tissue engineering in reconstruction of calvarial defects. J Biomed Mater Res A. 2011;96(3):543-51.

18. Kim HO, Kim E, An Y, et al. A biodegradable polymersome containing Bcl-xL siRNA and doxorubicin as a dual delivery vehicle for a synergistic anticancer effect. Macromol Biosci. 2013;13(6):745-754.

19. Cao N, Cheng D, Zou S, Ai H, Gao J, Shuai X. The synergistic effect of hierarchical assemblies of siRNA and chemotherapeutic drugs co-delivered into hepatic cancer cells. Biomaterials. 2011;32(8):2222-2232.
20. Singh A, Nie H, Ghosn B, Qin H, Kwak LW, Roy K. Efficient modulation of T-cell response by dual-mode, single-carrier delivery of cytokine-targeted siRNA and DNA vaccine to antigen-presenting cells. Mol Ther. 2008;16(12):2011-2021.

21. Oungbho K, Müller BW. Chitosan sponges as sustained release drug carriers. Int J Pharm. 1997;156:229-237.

22. Kinoshita Y, Maeda H. Recent developments of functional scaffolds for craniomaxillofacial bone tissue engineering applications. Scientific World Journal. 2013;2013:863157.

23. Wu K, Song W, Zhao L, et al. MicroRNA functionalized microporous titanium oxide surface by lyophilization with enhanced osteogenic activity. ACS Appl Mater Interfaces. 2013;5(7):2733-2744.

24. Yadava P, Gibbs M, Castro C, Hughes J. Effect of lyophilization and freeze-thawing on the stability of siRNA-liposome complexes. AAPS PharmSciTech. 2008;9(2):335-341.

25. Howard KA, Rahbek UL, Liu X, et al. RNA interference in vitro and in vivo using a chitosan/siRNA nanoparticle system. Mol Ther. 2006;14(4):476-484.

26. Andersen MO, Nygaard JV, Burns JS, et al. siRNA Nanoparticle functionalization of nanostructured scaffolds enables controlled multilineage differentiation of stem cells. Mol Ther. 2010;18(11): 2018-2027.

27. Yang YQ, Tan YY, Wong R, Wenden A, Zhang LK, Rabie ABM. The role of vascular endothelial growth factor in ossification. Int J Oral Sci. 2012;4(2):64-68.

28. Schipani E, Maes C, Carmeliet G, Semenza GL. Regulation of osteogenesis-angiogenesis coupling by HIFs and VEGF. J Bone Miner Res. 2009;24(8):1347-1353.
International Journal of Nanomedicine

\section{Publish your work in this journal}

The International Journal of Nanomedicine is an international, peerreviewed journal focusing on the application of nanotechnology in diagnostics, therapeutics, and drug delivery systems throughout the biomedical field. This journal is indexed on PubMed Central, MedLine, CAS, SciSearch $®$, Current Contents ${ }^{\circledR} /$ Clinical Medicine,

\section{Dovepress}

Journal Citation Reports/Science Edition, EMBase, Scopus and the Elsevier Bibliographic databases. The manuscript management system is completely online and includes a very quick and fair peer-review system, which is all easy to use. Visit http://www.dovepress.com/ testimonials.php to read real quotes from published authors. 